\title{
Microstructure Analysis of Thickness Effects in Thin Ferroelectric Polymer Film VDF/TrFE
}

\author{
Yuichiro Mabuchi ${ }^{1}$, Kaustav Chaudhuri ${ }^{2}$, James E. Evans ${ }^{3}$, Nigel D. Browning ${ }^{2,3,4}$, Soichiro \\ Okamura $^{1}$
}

${ }^{1}$ Department of Applied Physics, Faculty of Science, Tokyo University of Science, 1-3 Kagurazaka, Shinjuku-ku, Tokyo 162-8601

${ }^{2}$ Department of Chemical Engineering and Material Science, University of California, Davis, CA 95616

${ }^{3}$ Department of Molecular and Cell Biology, University of California, Davis CA95616

${ }^{4}$ Condensed Matter and Materials Division, Physical and Life Sciences Directorate, Lawrence Livermore National Laboratory, Livermore, CA 94550

Organic ferroelectrics may serve as ideal candidates for non-volatile memory applications because of their simplistic processing and cost-effectiveness [1]-[2]. One such organic ferroelectric is vinylidene fluoride / trifluoroethylene (VDF/TrFE), which is a complex copolymer consisting of both amorphous and crystalline phases [3]-[5]. For this set of experiments investigating the microstructure of the system, a thin film of the polymer was deposited on a substrate consisting of silicon, silica, titanium and platinum layers. A gold nanolayer was then deposited on top of the polymer which serves as an electrode. Annealing these layered samples at $120^{\circ} \mathrm{C}$ and $140^{\circ} \mathrm{C}$ orders the polymer thereby increasing its crystallinity, which in turn increases the ferroelectric nature of these samples. The goal of this study is to investigate the origin of the degradation of ferroelectric properties in thin polymer films. It is observed that the polarization from a hysteresis curve of the samples is depends on the thickness of the polymer film. One hypothesis is that the formation of an interface layer between the electrode and the polymer film prevents polarization reversal in thin polymer films more than thick polymer films. The basis for this claim is that the interface layer interrupts and reduces the electric field that is directed towards the polymer. Thus the key to understanding this problem is to analyze this interface layer and know its composition.

The complexity of the interfaces in this system mean that complete characterization of the structure requires a combination of both high resolution transmission electron microscopy (HRTEM) and scanning transmission electron microscopy (STEM). Using these two techniques together, the quality of the interfaces can be ascertained and the overall polymer thickness measured. Furthermore, using electron energy loss spectroscopy (EELS) can reveal the true identity of the interface layer. In order to perform TEM/STEM analyses, the sample has to be thinned down so that it is electron transparent. A focused ion beam (FIB) has been used to lift-out and thin down a cross section of the sample. Two protective platinum layers have been added on top of the gold and the polymer to prevent any kind of damage during the process. One was deposited as a thick ion beam layer and the other as a thin electron beam layer. Figure 1 shows a thinned sample annealed at $140^{\circ} \mathrm{C}$ with all the layers present. The higher magnification image in Figure 2 indicates the quality of the polymer and allows it to be measured to be $21.45 \pm 3.84 \mathrm{~nm}$. In this presentation, results from the three types of samples prepared under different conditions will be shown and the implications of the microstructure in determining the overall properties of the system will be discussed [6]. 


\section{References}

[1] D. Y. Kusuma et al., J. Phys. Chem. B 114 (2010) 13289.

[2] R.G. C. Naber et al., Adv. Mater. 21 (2009) 1.

[3] T. Nakajima et al., Jpn. J. Appl. Phys. 48 (2009) 09KE04

[4] M. J. Bai et al., J. Phys. Condens. Matter. 19 (2007) 196211

[5] Nalwa, H. S. Handbook of thin film materials, Vol. 3: Ferroelectric and Dielectric Thin Films; Academic Press, New York, 2002.

[6] This work is supported by the University of California through the UC Lab Fee Research Program.

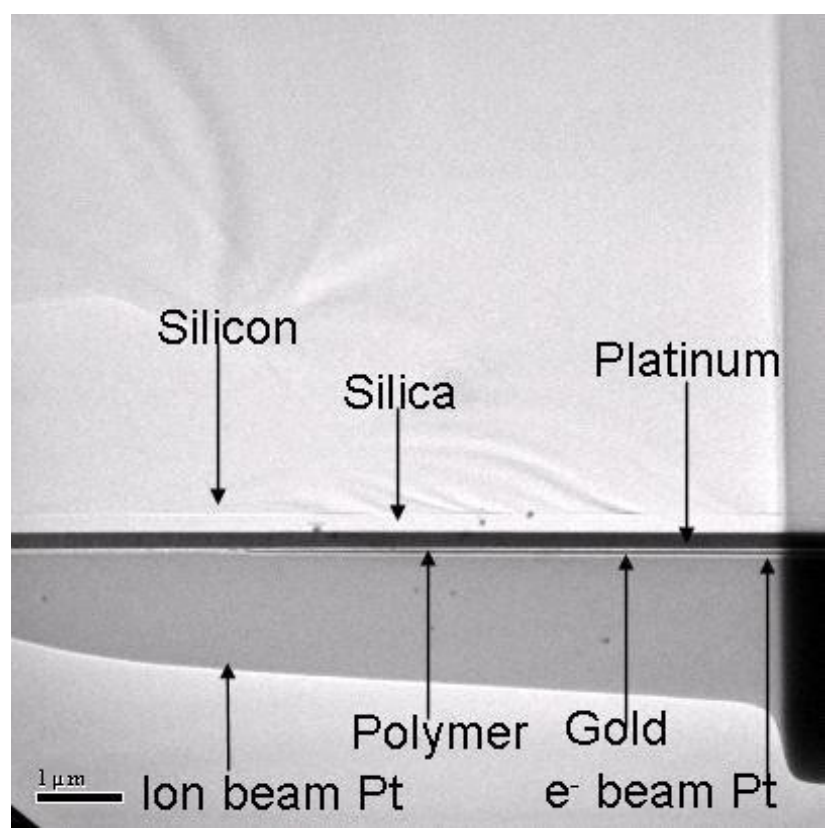

FIG. 1.TEM images showing a FIB sample annealed at $140^{\circ} \mathrm{C}$ with the different layers.

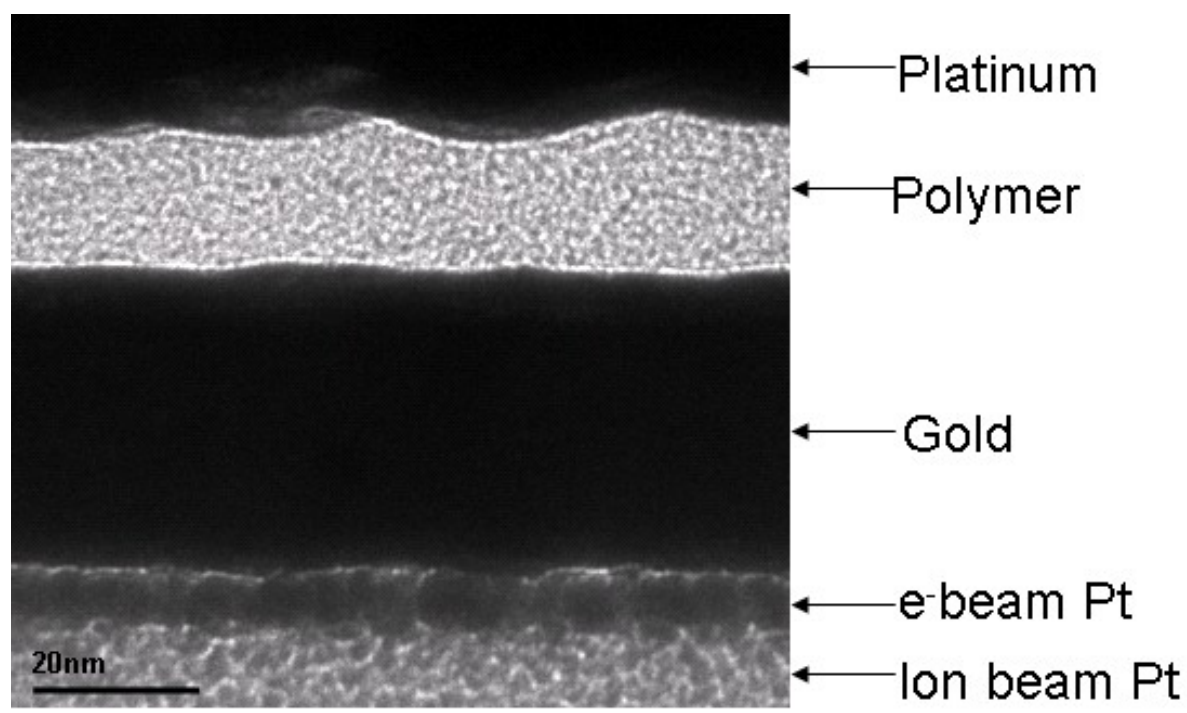

FIG. 2. TEM image showing different layers of interest at a magnification of $800 \mathrm{KX}$ 